\title{
Erratum: Nonparametric Estimation about Species not Observed in a Random Sample
}

\author{
Alberto Gandolfi and C.C.A. Sastri
}

In [1], page 91 , line 2 should be changed to

$$
\hat{C}_{A C E}=1-n_{1} / n_{\text {rare }} .
$$

page 96 line 4 should be changed to

$$
E_{T}=\frac{T-N}{\left(n_{1}+T-N\right)} \frac{\left(2 n_{1}+T-N\right)}{(T+n)} .
$$

page 96 lines 10-13 should be changed to

therefore, rather surprisingly, $\hat{T}_{G, S}^{\prime}=\hat{T}_{C L}(1)$, which is to say that our first estimator equals the Chao-Lee estimator with their estimated variance equal to 1 . Although...

pages 101-102, Tables 6.1 and 6.2 , row 2 column 1 should be changed to

$$
T_{T G}
$$

\section{References}

[1] Alberto Gandolfi, C.C.A. Sastri, Nonparametric Estimation about Species not Observed in a Random Sample, Milan j. math. 72 (2004), 81-105. 
Alberto Gandolfi

Dipartimento di Matematica U. Dini,

Università di Firenze,

Viale Morgagni 67/A, 50134 Firenze, Italy

e-mail: gandolfi@math.unifi.it

C.C.A. Sastri

Department of Mathematics and Statistics

Dalhousie University, Halifax, Nova Scotia Canada B3H 3J5

e-mail: sastri@mathstat.dal.ca

Do Toccess this journal online:

(4) http://www.birkhauser.ch 